\title{
GENERALIZATIONS OF A WEIGHTED TRAPEZOIDAL INEQUALITY FOR MONOTONIC FUNCTIONS AND APPLICATIONS
}

\author{
KUEI-LIN TSENG ${ }^{1}$, GOU-SHENG YANG ${ }^{2}$ and SEVER S. DRAGOMIR ${ }^{\Xi}$
}

(Received 10 February 2006)

\begin{abstract}
In this paper we establish some generalizations of a weighted trapezoidal inequality for monotonic functions and give several applications for the $r$-moments, the expectation of a continuous random variable and the Beta and Gamma functions.

2000 Mathematics subject classification: primary 26D15; secondary 41A55.

Keywords and phrases: trapezoidal inequality, monotonic functions, $r$-moments, expectation of a continuous random variable, the Beta and Gamma functions.
\end{abstract}

\section{Introduction}

The trapezoidal inequality states that if $f^{\prime \prime}$ exists and is bounded on $(a, b)$, then

$$
\left|\int_{a}^{b} f(x) d x-\frac{b-a}{2}[f(a)+f(b)]\right| \leq \frac{(b-a)^{3}}{12}\left\|f^{\prime \prime}\right\|_{\infty},
$$

where $\left\|f^{\prime \prime}\right\|_{\infty}:=\sup _{x \in(a, b)}\left|f^{\prime \prime}(x)\right|<\infty$.

Now if we assume that $I_{n}: a=x_{0}<x_{1}<\cdots<x_{n}=b$ is a partition of the interval $[a, b]$ and $f$ is as above, then we can approximate the integral $\int_{a}^{b} f(x) d x$ by the trapezoidal quadrature rule $A_{T}\left(f, I_{n}\right)$, having an error denoted by $R_{T}\left(f, I_{n}\right)$, where

$$
A_{T}\left(f, I_{n}\right):=\frac{1}{2} \sum_{i=0}^{n-1}\left[f\left(x_{i}\right)+f\left(x_{i+1}\right)\right] l_{i}, .
$$

\footnotetext{
${ }^{1}$ Department of Mathematics, Aletheia University, Tamsui 25103, Taiwan; e-mail: kltseng@email.au.edu.tw.

${ }^{2}$ Department of Mathematics, Tamkang University, Tamsui 25137, Taiwan.

${ }^{3}$ School of Computer Science and Mathematics, Victoria University, PO Box 14428, MCMC 8001, Victoria, Australia; e-mail; sever.dragomir@vu.edu.au.

(C) Australian Mathematical Society 2007, Serial-fee code 1446-1811/07
} 
and the remainder $R_{T}\left(f, I_{n}\right)$ satisfies the estimation

$$
\left|R_{T}\left(f, I_{n}\right)\right| \leq \frac{1}{12}\left\|f^{\prime \prime}\right\|_{\infty} \sum_{i=0}^{n-1} l_{i}^{3},
$$

with $l_{i}:=x_{i+1}-x_{i}$ for $i=0,1, \ldots, n-1$.

For some recent results which generalize, improve and extend this classic inequality (1.1), see the papers [2-8].

Recently, Cerone and Dragomir [3] proved the following two trapezoidal-type inequalities for monotonic functions:

THEOREM A. Let $f:[a, b] \rightarrow \mathbb{B}$ be a monotonic nondecreasing function on $[a, b]$, then

$$
\begin{aligned}
& \left|\int_{a}^{b} f(t) d t-[(x-a) f(a)+(b-x) f(b)]\right| \\
& \quad \leq(b-x) f(b)-(x-a) f(a)+\int_{a}^{b} \operatorname{sgn}(x-t) f(t) d t \\
& \quad \leq(x-a)[f(x)-f(a)]+(b-x)[f(b)-f(x)] \\
& \quad \leq\left[\frac{1}{2}(b-a)+\left|x-\frac{a+b}{2}\right|\right][f(b)-f(a)],
\end{aligned}
$$

for all $x \in[a, b]$. The above inequalities are sharp.

Let $I_{n}, l_{i}(i=0,1, \ldots, n-1)$ be as above and let $\xi_{t} \in\left[x_{i}, x_{i+1}\right](i=0,1, \ldots, n-1)$ be intermediate points. Define the generalized trapezoidal quadrature rule by

$$
T_{P}\left(f, I_{n}, \xi\right):=\sum_{i=0}^{n-1}\left[\left(\xi_{i}-x_{i}\right) f\left(x_{i}\right)+\left(x_{i+1}-\xi_{i}\right) f\left(x_{i+1}\right)\right] .
$$

We have the following result for the approximation of $\int_{a}^{b} f(x) d x$ in terms of $T_{P}$.

THEOREM B. Let $f$ be defined as in Theorem A, then we have

$$
\int_{a}^{b} f(x) d x=T_{P}\left(f, I_{n}, \xi\right)+R_{P}\left(f, I_{n}, \xi\right) .
$$

The remainder term $\dot{R}_{P}\left(f, I_{n}, \xi\right)$ satisfies the inequalities

$$
\begin{aligned}
\left|R_{P}\left(f, I_{n}, \xi\right)\right| & \leq \sum_{i=0}^{n-1}\left[\left(x_{i+1}-\xi_{i}\right) f\left(x_{i+1}\right)-\left(\xi_{i}-x_{i}\right) f\left(x_{i}\right)\right]+\sum_{i=0}^{n-1} \int_{x_{i}}^{x_{i+1}} \operatorname{sgn}\left(\xi_{i}-t\right) f(t) d t \\
& \leq \sum_{i=0}^{n-1}\left(\xi_{i}-x_{i}\right)\left[f\left(\xi_{i}\right)-f\left(x_{i}\right)\right]+\sum_{i=0}^{n-1}\left(x_{i+1}-\xi_{i}\right)\left[f\left(x_{i+1}\right)-f\left(\xi_{i}\right)\right]
\end{aligned}
$$




$$
\begin{aligned}
& \leq \sum_{i=0}^{n-1}\left[\frac{1}{2} l_{i}+\left|\xi_{i}-\frac{x_{i}+x_{i+1}}{2}\right|\right]\left[f\left(x_{i+1}\right)-f\left(x_{i}\right)\right] \\
& \leq\left[\frac{1}{2} \nu(l)+\max _{i=0,1, \ldots, n-1}\left|\xi_{i}-\frac{x_{i}+x_{i+1}}{2}\right|\right][f(b)-f(a)] \\
& \leq v(l)[f(b)-f(a)],
\end{aligned}
$$

where $v(l):=\max \left\{l_{i} \mid i=0,1, \ldots, n-1\right\}$.

In this paper we establish weighted generalizations of Theorems A and B and give several applications for the $r$-moments, the expectation of a continuous random variable and the Beta and Gamma functions.

\section{Some integral inequalities}

The following result shows a generalization of the weighted trapezoidal inequality.

THEOREM 1. Let $g:[a, b] \rightarrow \mathbb{R}$ be nonnegative and continuous with $g(t)>0$ on $(a, b)$ and let $h:[a, b] \rightarrow \mathbb{B}$ be differentiable such that $h^{\prime}(t)=g(t)$ on $[a, b]$.

(a) If $f:[a, b] \rightarrow \mathbb{R}$ is a monotonic nondecreasing function on $[a, b]$, then

$$
\begin{aligned}
& \left|\int_{a}^{b} f(t) g(t) d t-[(x-h(a)) f(a)+(h(b)-x) f(b)]\right| \\
& \quad \leq(h(b)-x) f(b)-(x-h(a)) f(a)+\int_{a}^{b} \operatorname{sgn}\left(h^{-1}(x)-t\right) f(t) g(t) d t \\
& \quad \leq(x-h(a))\left[f\left(h^{-1}(x)\right)-f(a)\right]+(h(b)-x)\left[f(b)-f\left(h^{-1}(x)\right)\right] \\
& \quad \leq\left[\frac{1}{2} \int_{a}^{b} g(t) d t+\left|x-\frac{h(a)+h(b)}{2}\right|\right][f(b)-f(a)]
\end{aligned}
$$

for all $x \in[h(a), h(b)]$.

(b) If $f:[a, b] \rightarrow \mathbb{R}$ is a monotonic nonincreasing function on $[a, b]$, then

$$
\begin{aligned}
& \left|\int_{a}^{b} f(t) g(t) d t-[(x-h(a)) f(a)+(h(b)-x) f(b)]\right| \\
& \quad \leq(x-h(a)) f(a)-(h(b)-x) f(b)+\int_{a}^{b} \operatorname{sgn}\left(t-h^{-1}(x)\right) f(t) g(t) d t \\
& \quad \leq(x-h(a))\left[f(a)-f\left(h^{-1}(x)\right)\right]+(h(b)-x)\left[f\left(h^{-1}(x)\right)-f(b)\right] \\
& \quad \leq\left[\frac{1}{2} \int_{a}^{b} g(t) d t+\left|x-\frac{h(a)+h(b)}{2}\right|\right][f(a)-f(b)]
\end{aligned}
$$

for all $x \in[h(a), h(b)]$.

The above inequalities are sharp. 
PROOF. (a) Let $x \in[h(a), h(b)]$. Using the integration by parts formula for the Riemann-Stieltjes integral, we have the following identity:

$$
\begin{aligned}
\int_{a}^{b}(x-h(t)) d f(t) & =\left.(x-h(t)) f(t)\right|_{a} ^{b}+\int_{a}^{b} f(t) g(t) d t \\
& =\int_{a}^{b} f(t) g(t) d t-[(x-h(a)) f(a)+(h(b)-x) f(b)] .
\end{aligned}
$$

It is well known [1, page 813] that if $\mu, \nu:[a, b] \rightarrow \mathbb{R}$ are such that $\mu$ is continuous on $[a, b]$ and $\nu$ is monotonic nondecreasing on $[a, b]$, then

$$
\left|\int_{a}^{b} \mu(t) d \nu(t)\right| \leq \int_{a}^{b}|\mu(t)| d \nu(t) .
$$

Now, using identity (2.3) and inequality (2.4), we have

$$
\begin{aligned}
\left|\int_{a}^{b} f(t) g(t) d t-[(x-h(a)) f(a)+(h(b)-x) f(b)]\right| \\
\leq \int_{a}^{b}|x-h(t)| d f(t) \\
=\int_{a}^{h^{-1}(x)}(x-h(t)) d f(t)+\int_{h^{-1}(x)}^{b}(h(t)-x) d f(t) \\
=\left.(x-h(t)) f(t)\right|_{a} ^{h^{-1}(x)}+\int_{a}^{h^{-1}(x)} f(t) g(t) d t \\
\quad+\left.(h(t)-x)\right|_{h^{-1}(x)} ^{b}-\int_{h^{-1}(x)}^{b} f(t) g(t) d t \\
=(h(b)-x) f(b)-(x-h(a)) f(a)+\int_{a}^{b} \operatorname{sgn}\left(h^{-1}(x)-t\right) f(t) g(t) d t
\end{aligned}
$$

and the first inequalities in (2.1) are proved.

As $f$ is monotonic nondecreasing on $[a, b]$, we obtain

$$
\int_{a}^{h^{-1}(x)} f(t) g(t) d t \leq f\left(h^{-1}(x)\right) \int_{a}^{h^{-1}(x)} g(t) d t=(x-h(a)) f\left(h^{-1}(x)\right)
$$

and

$$
\int_{h^{-1}(x)}^{b} f(t) g(t) d t \geq f\left(h^{-1}(x)\right) \int_{h^{-1}(x)}^{b} g(t) d t=(h(b)-x) f\left(h^{-1}(x)\right),
$$

then

$$
\int_{a}^{b} \operatorname{sgn}\left(h^{-1}(x)-t\right) f(t) g(t) d t \leq(x-h(a)) f\left(h^{-1}(x)\right)+(x-h(b)) f\left(h^{-1}(x)\right) .
$$


Therefore

$$
\begin{aligned}
(h(b)- & x) f(b)-(x-h(a)) f(a)+\int_{a}^{b} \operatorname{sgn}\left(h^{-1}(x)-t\right) f(t) g(t) d t \\
\leq & (h(b)-x) f(b)-(x-h(a)) f(a) \\
& +(x-h(a)) f\left(h^{-1}(x)\right)+(x-h(b)) f\left(h^{-1}(x)\right) \\
= & (x-h(a))\left[f\left(h^{-1}(x)\right)-f(a)\right]+(h(b)-x)\left[f(b)-f\left(h^{-1}(x)\right)\right],
\end{aligned}
$$

which proves the second inequality in (2.1).

As $f$ is monotonic nondecreasing on $[a, b]$, we have $f(a) \leq f\left(h^{-1}(x)\right) \leq f(b)$ and

$$
\begin{aligned}
(x- & h(a))\left[f\left(h^{-1}(x)\right)-f(a)\right]+(h(b)-x)\left[f(b)-f\left(h^{-1}(x)\right)\right] \\
& \leq \max \{x-h(a), h(b)-x\}\left[f\left(h^{-1}(x)\right)-f(a)+f(b)-f\left(h^{-1}(x)\right)\right] \\
& =\left[\frac{h(b)-h(a)}{2}+\left|x-\frac{h(a)+h(b)}{2}\right|\right][f(b)-f(a)] \\
& =\left[\frac{1}{2} \int_{a}^{b} g(t) d t+\left|x-\frac{h(a)+h(b)}{2}\right|\right][f(b)-f(a)] .
\end{aligned}
$$

Thus, by (2.5)-(2.7), we obtain (2.1).

Let $g(t) \equiv 1, h(t)=t, t \in[a, b]$;

$$
f(t)= \begin{cases}0, & t \in[a, b), \\ 1, & t=b\end{cases}
$$

and $x=(a+b) / 2$. Then a simple calculation reveals that

$$
\begin{aligned}
& \left|\int_{a}^{b} f(t) g(t) d t-[(x-h(a)) f(a)+(h(b)-x) f(b)]\right| \\
& \quad=(h(b)-x) f(b)-(x-h(a)) f(a)+\int_{a}^{b} \operatorname{sgn}\left(h^{-1}(x)-t\right) f(t) g(t) d t \\
& \quad=(x-h(a))\left[f\left(h^{-1}(x)\right)-f(a)\right]+(h(b)-x)\left[f(b)-f\left(h^{-1}(x)\right)\right] \\
& \quad=\left[\frac{1}{2} \int_{a}^{b} g(t) d t+\left|x-\frac{h(a)+h(b)}{2}\right|\right][f(b)-f(a)] \\
& \quad=\frac{b-a}{2},
\end{aligned}
$$

which proves that the inequalities (2.1) are sharp.

(b) If $f$ is replaced by $-f$ in (a), then (2.2) is obtained from (2.1). This completes the proof. 
REMARK 1. If we choose $g(t) \equiv 1$ and $h(t)=t$ on $[a, b]$, then inequalities (2.1) reduce to (1.2).

COROLLARY 1. If we choose $x=(h(a)+h(b)) / 2$, then we get

$$
\begin{aligned}
\left|\int_{a}^{b} f(t) g(t) d t-\frac{f(a)+f(b)}{2} \int_{a}^{b} g(t) d t\right| \\
\leq \leq \frac{1}{2}[f(b)-f(a)] \int_{a}^{b} g(t) d t \\
\quad+\int_{a}^{b} \operatorname{sgn}\left(h^{-1}\left(\frac{h(a)+h(b)}{2}\right)-t\right) f(t) g(t) d t \\
\leq \frac{1}{2}[f(b)-f(a)] \int_{a}^{b} g(t) d t,
\end{aligned}
$$

where $f$ and $g$ are defined as in (a) of Theorem 1, and

$$
\begin{aligned}
\left|\int_{a}^{b} f(t) g(t) d t-\frac{f(a)+f(b)}{2} \int_{a}^{b} g(t) d t\right| \\
\leq \frac{1}{2}[f(a)-f(b)] \int_{a}^{b} g(t) d t \\
\quad+\int_{a}^{b} \operatorname{sgn}\left(t-h^{-1}\left(\frac{h(a)+h(b)}{2}\right)\right) f(t) g(t) d t \\
\leq \leq \frac{1}{2}[f(a)-f(b)] \int_{a}^{b} g(t) d t,
\end{aligned}
$$

where $f$ and $g$ are defined as in (b) of Theorem 1 .

The inequalities (2.8) and (2.9) are the "weighted trapezoid" inequalities.

Note that the trapezoidal inequalities (2.8) and (2.9) are, in a sense, the best possible inequalities we can obtain from (2.1) and (2.2). Moreover, the constant $1 / 2$ is the best possible for both inequalities in (2.8) and (2.9), respectively.

REMARK 2. The following inequality is well known in the literature as the Fejér inequality (see for example [9]):

$$
f\left(\frac{a+b}{2}\right) \int_{a}^{b} g(t) d t \leq \int_{a}^{b} f(t) g(t) d t \leq \frac{f(a)+f(b)}{2} \int_{a}^{b} g(t) d t,
$$

where $f:[a, b] \rightarrow \mathbb{R}$ is convex and $g:[a, b] \rightarrow \mathbb{R}$ is positive, integrable and symmetric to $(a+b) / 2$. 
Using the above results and (2.8)-(2.9), we obtain the following error bound of the second inequality in (2.10):

$$
\begin{aligned}
0 \leq & \frac{f(a)+f(b)}{2} \int_{a}^{b} g(t) d t-\int_{a}^{b} f(t) g(t) d t \\
\leq & \frac{1}{2}[f(b)-f(a)] \int_{a}^{b} g(t) d t \\
& +\int_{a}^{b} \operatorname{sgn}\left(h^{-1}\left(\frac{h(a)+h(b)}{2}\right)-t\right) f(t) g(t) d t \\
\leq & \frac{1}{2}[f(b)-f(a)] \int_{a}^{b} g(t) d t,
\end{aligned}
$$

provided that $f$ is monotonic nondecreasing on $[a, b]$.

Also,

$$
\begin{aligned}
0 & \leq \frac{f(a)+f(b)}{2} \int_{a}^{b} g(t) d t-\int_{a}^{b} f(t) g(t) d t \\
& \leq \frac{1}{2}[f(a)-f(b)] \int_{a}^{b} g(t) d t+\int_{a}^{b} \operatorname{sgn}\left(t-h^{-1}\left(\frac{h(a)+h(b)}{2}\right)\right) f(t) g(t) d t \\
& \leq \frac{1}{2}[f(a)-f(b)] \int_{a}^{b} g(t) d t
\end{aligned}
$$

provided that $f$ is monotonic nonincreasing on $[a, b]$.

\section{Applications for the quadrature rules}

Throughout this section, let $g$ and $h$ be defined as in Theorem 1 .

Let $f:[a, b] \rightarrow \mathbb{R}$, and let $I_{n}: a=x_{0}<x_{1}<\cdots<x_{n}=b$ be a partition of $[a, b]$ and $\xi_{i} \in\left[h\left(x_{i}\right), h\left(x_{t+1}\right)\right](i=0,1, \ldots, n-1)$ be intermediate points. Put $l_{l}:=h\left(x_{t+1}\right)-h\left(x_{i}\right)=\int_{x_{i}}^{x_{i+1}} g(t) d t$ and define the quadrature rule

$$
T_{P}\left(f, g, h, I_{n}, \xi\right):=\sum_{i=0}^{n-1}\left[\left(\xi_{i}-h\left(x_{i}\right)\right) f\left(x_{i}\right)+\left(h\left(x_{i+1}\right)-\xi_{i}\right) f\left(x_{i+1}\right)\right] .
$$

We have the following result concerning the approximation of $\int_{a}^{b} f(t) g(t) d t$ in terms of $T_{P}$.

THEOREM 2. Let $v(l):=\max \left\{l_{\imath} \mid i=0,1, \ldots, n-1\right\}, f$ be defined as in Theorem 1 and let

$$
\int_{a}^{b} f(t) g(t) d t=T_{P}\left(f, g, h, I_{n}, \xi\right)+R_{P}\left(f, g, h, I_{n}, \xi\right) .
$$


(a) If $f$ is monotonic nondecreasing on $[a, b]$, then

$$
\begin{aligned}
&\left|R_{P}\left(f, g, h, I_{n}, \xi\right)\right| \\
& \leq \sum_{t=0}^{n-1}\left[\left(h\left(x_{i+1}\right)-\xi_{i}\right) f\left(x_{i+1}\right)-\left(\xi_{i}-h\left(x_{i}\right)\right) f\left(x_{i}\right)\right] \\
& \quad+\sum_{i=0}^{n-1} \int_{x_{i}}^{x_{t+1}} \operatorname{sgn}\left(h^{-1}\left(\xi_{i}\right)-t\right) f(t) g(t) d t \\
& \leq \sum_{i=0}^{n-1}\left(\xi_{i}-h\left(x_{i}\right)\right)\left[f\left(h^{-1}\left(\xi_{i}\right)\right)-f\left(x_{t}\right)\right] \\
&+\sum_{i=0}^{n-1}\left(h\left(x_{i+1}\right)-\xi_{i}\right)\left[f\left(x_{i+1}\right)-f\left(h^{-1}\left(\xi_{i}\right)\right)\right] \\
& \leq \sum_{i=0}^{n-1}\left[\frac{1}{2} l_{i}+\left|\xi_{i}-\frac{h\left(x_{i}\right)+h\left(x_{i+1}\right)}{2}\right|\right]\left[f\left(x_{i+1}\right)-f\left(x_{i}\right)\right] \\
& \leq {\left[\frac{1}{2} v(l)+\max _{i=0,1, \ldots n-1}\left|\xi_{i}-\frac{h\left(x_{i}\right)+h\left(x_{i+1}\right)}{2}\right|\right][f(b)-f(a)] } \\
& \leq v(l)[f(b)-f(a)] .
\end{aligned}
$$

(b) If $f$ is monotonic nonincreasing on $[a, b]$, then

$$
\begin{aligned}
\left|R_{P}\left(f, g, h, I_{n}, \xi\right)\right| & \\
\leq & \sum_{i=0}^{n-1}\left[\left(\xi_{i}-h\left(x_{i}\right)\right) f\left(x_{i}\right)-\left(h\left(x_{i+1}\right)-\xi_{i}\right) f\left(x_{i+1}\right)\right] \\
& +\sum_{i=0}^{n-1} \int_{x_{i}}^{x_{i+1}} \operatorname{sgn}\left(t-h^{-1}\left(\xi_{i}\right)\right) f(t) g(t) d t \\
\leq & \sum_{i=0}^{n-1}\left(\xi_{i}-h\left(x_{i}\right)\right)\left[f\left(x_{i}\right)-f\left(h^{-1}\left(\xi_{i}\right)\right)\right] \\
& +\sum_{i=0}^{n-1}\left(h\left(x_{i+1}\right)-\xi_{i}\right)\left[f\left(h^{-1}\left(\xi_{i}\right)\right)-f\left(x_{i+1}\right)\right] \\
\leq & \sum_{i=0}^{n-1}\left[\frac{1}{2} l_{i}+\left|\xi_{i}-\frac{h\left(x_{i}\right)+h\left(x_{i+1}\right)}{2}\right|\right]\left[f\left(x_{i}\right)-f\left(x_{i+1}\right)\right] \\
\leq & {\left[\frac{1}{2} v(l)+\max _{i=0.1, \ldots, n-1}\left|\xi_{i}-\frac{h\left(x_{i}\right)+h\left(x_{i+1}\right)}{2}\right|\right][f(a)-f(b)] } \\
\leq & v(l)[f(a)-f(b)] .
\end{aligned}
$$


PROOF. (a) Apply Theorem 1 on the intervals $\left[x_{i}, x_{i+1}\right](i=0,1, \ldots, n-1)$ to get

$$
\begin{array}{rl}
\mid \int_{x_{i}}^{x_{i+1}} & f(t) g(t) d t-\left[\left(\xi_{i}-h\left(x_{i}\right)\right) f\left(x_{i}\right)+\left(h\left(x_{i+1}\right)-\xi_{i}\right) f\left(x_{i+1}\right)\right] \mid \\
\leq & \left(h\left(x_{i+1}\right)-\xi_{i}\right) f\left(x_{i+1}\right)-\left(\xi_{i}-h\left(x_{i}\right)\right) f\left(x_{i}\right) \\
& \quad+\int_{x_{i}}^{x_{i+1}} \operatorname{sgn}\left(h^{-1}\left(\xi_{i}\right)-t\right) f(t) g(t) d t \\
\leq & \left(\xi_{i}-h\left(x_{i}\right)\right)\left[f\left(h^{-1}\left(\xi_{i}\right)\right)-f\left(x_{i}\right)\right] \\
& +\left(h\left(x_{i+1}\right)-\xi_{i}\right)\left[f\left(x_{i+1}\right)-f\left(h^{-1}\left(\xi_{i}\right)\right)\right] \\
\leq & {\left[\frac{1}{2} l_{i}+\left|\xi_{i}-\frac{h\left(x_{i}\right)+h\left(x_{i+1}\right)}{2}\right|\right]\left[f\left(x_{i+1}\right)-f\left(x_{i}\right)\right]}
\end{array}
$$

for all $i \in\{0,1, \ldots, n-1\}$.

Using this and the generalized triangle inequality, we have

$$
\begin{aligned}
\mid R_{P}( & \left.f, g, h, I_{n}, \xi\right) \mid \\
\leq & \sum_{i=0}^{n-1}\left|\int_{x_{i}}^{x_{i+1}} f(t) g(t) d t-\left[\left(\xi_{i}-h\left(x_{i}\right)\right) f\left(x_{i}\right)+\left(h\left(x_{i+1}\right)-\xi_{i}\right) f\left(x_{i+1}\right)\right]\right| \\
\leq & \sum_{i=0}^{n-1}\left[\left(h\left(x_{i+1}\right)-\xi_{i}\right) f\left(x_{i+1}\right)-\left(\xi_{i}-h\left(x_{i}\right)\right) f\left(x_{i}\right)\right] \\
& +\sum_{i=0}^{n-1} \int_{x_{i}}^{x_{i+1}} \operatorname{sgn}\left(h^{-1}\left(\xi_{i}\right)-t\right) f(t) g(t) d t \\
\leq & \sum_{i=0}^{n-1}\left(\xi_{i}-h\left(x_{i}\right)\right)\left[f\left(h^{-1}\left(\xi_{i}\right)\right)-f\left(x_{i}\right)\right] \\
& +\sum_{i=0}^{n-1}\left(h\left(x_{i+1}\right)-\xi_{i}\right)\left[f\left(x_{i+1}\right)-f\left(h^{-1}\left(\xi_{i}\right)\right)\right] \\
\leq & \sum_{i=0}^{n-1}\left[\frac{1}{2} l_{i}+\left|\xi_{i}-\frac{h\left(x_{i}\right)+h\left(x_{i+1}\right)}{2}\right|\right]\left[f\left(x_{i+1}\right)-f\left(x_{i}\right)\right] \\
\leq & {\left[\frac{1}{2} \nu(l)+\max _{i=0,1, \ldots, n-1}\left|\xi_{i}-\frac{h\left(x_{i}\right)+h\left(x_{i+1}\right)}{2}\right|\right][f(b)-f(a)] . }
\end{aligned}
$$

Next, observe that

$$
\left|\xi_{i}-\frac{h\left(x_{i}\right)+h\left(x_{i+1}\right)}{2}\right| \leq \frac{1}{2} l_{i} \quad(i=0,1, \ldots, n-1)
$$


and then

$$
\max _{i=0,1, \ldots, n-1}\left|\xi_{i}-\frac{h\left(x_{i}\right)+h\left(x_{i+1}\right)}{2}\right| \leq \frac{1}{2} \nu(l) .
$$

Thus, by (3.3)-(3.5), we obtain (3.2).

(b) The proof is similar to that for (a) and we omit the details.

REMARK 3. If we choose $g(t) \equiv 1, h(t)=t$ on $[a, b]$, then the inequalities (3.2) reduce to (1.3).

Now, let $\xi_{i}=\left(h\left(x_{i}\right)+h\left(x_{i+1}\right)\right) / 2(i=0,1, \ldots, n-1)$ and let $T_{P W}\left(f, g, h, I_{n}\right)$ and $R_{P}\left(f, g, h, I_{n}\right)$ be defined as

$$
T_{P W}\left(f, g, h, I_{n}\right)=T_{P}\left(f, g, h, I_{n}, \xi\right)=\frac{1}{2} \sum_{i=0}^{n-1}\left[f\left(x_{i}\right)+f\left(x_{i+1}\right)\right] \int_{x_{i}}^{x_{i+1}} g(t) d t
$$

and

$$
\begin{aligned}
R_{P W}\left(f, g, h, I_{n}\right) & =R_{P}\left(f, g, h, I_{n}, \xi\right) \\
& =\int_{a}^{b} f(t) g(t) d t-\frac{1}{2} \sum_{i=0}^{n-1}\left[f\left(x_{i}\right)+f\left(x_{i+1}\right)\right] \int_{x_{i}}^{x_{t+1}} g(t) d t .
\end{aligned}
$$

If we consider the weighted trapezoidal formula $T_{P W}\left(f, g, h, I_{n}\right)$, then we have the following corollary.

COROLlaRY 2. Let $f, g, h$ be defined as in Theorem 2 and $\xi_{i}=\left(h\left(x_{i}\right)+h\left(x_{i+1}\right)\right) / 2$ $(i=0,1, \ldots, n-1)$. Then

$$
\int_{a}^{b} f(t) g(t) d t=T_{P W}\left(f, g, h, I_{n}\right)+R_{P W}\left(f, g, h, I_{n}\right)
$$

where the remainder satisfies the following estimates:

(a) If $f$ is monotonic nondecreasing on $[a, b]$, then

$$
\begin{aligned}
\left|R_{P W}\left(f, g, h, I_{n}\right)\right| \leq & \frac{1}{2} \sum_{i=0}^{n-1}\left(\int_{x_{i}}^{x_{i+1}} g(t) d t\right)\left[f\left(x_{i+1}\right)-f\left(x_{i}\right)\right] \\
& +\sum_{i=0}^{n-1} \int_{x_{i}}^{x_{i+1}} \operatorname{sgn}\left(h^{-1}\left(\frac{h\left(x_{i}\right)+h\left(x_{i+1}\right)}{2}\right)-t\right) f(t) g(t) d t \\
\leq & \frac{1}{2} \sum_{i=0}^{n-1}\left(\int_{x_{i}}^{x_{i+1}} g(t) d t\right)\left[f\left(x_{i+1}\right)-f\left(x_{i}\right)\right] \\
\leq & \frac{\nu(l)}{2}[f(b)-f(a)] .
\end{aligned}
$$


(b) If $f$ is monotonic nonincreasing on $[a, b]$, then

$$
\begin{aligned}
\left|R_{P W}\left(f, g, h, I_{n}\right)\right| \leq & \frac{1}{2} \sum_{i=0}^{n-1}\left(\int_{x_{i}}^{x_{i+1}} g(t) d t\right)\left[f\left(x_{i}\right)-f\left(x_{i+1}\right)\right] \\
& +\sum_{i=0}^{n-1} \int_{x_{i}}^{x_{i+1}} \operatorname{sgn}\left(t-h^{-1}\left(\frac{h\left(x_{i}\right)+h\left(x_{i+1}\right)}{2}\right)\right) f(t) g(t) d t \\
\leq & \frac{1}{2} \sum_{i=0}^{n-1}\left(\int_{x_{i}}^{x_{i+1}} g(t) d t\right)\left[f\left(x_{i}\right)-f\left(x_{i+1}\right)\right] \\
\leq & \frac{v(l)}{2}[f(a)-f(b)] .
\end{aligned}
$$

REMARK 4. In Corollary 2, suppose $f$ is monotonic on $[a, b]$,

$$
x_{t}=h^{-1}\left[h(a)+\frac{i(h(b)-h(a))}{n}\right] \quad(i=0,1, \ldots, n),
$$

and

$$
l_{i}:=h\left(x_{i+1}\right)-h\left(x_{i}\right)=\frac{h(b)-h(a)}{n}=\frac{1}{n} \int_{a}^{b} g(t) d t . \quad(i=0,1, \ldots, n-1) .
$$

If we want to approximate the integral $\int_{a}^{b} f(t) g(t) d t$ by $T_{P W}\left(f, g, h, I_{n}\right)$ with an accuracy greater than $\varepsilon>0$, we need at least $n_{\varepsilon} \in \mathbb{N}$ points for the partition $I_{n}$, where

$$
n_{\varepsilon}:=\left[\frac{1}{2 \varepsilon} \int_{a}^{b} g(t) d t \cdot|f(b)-f(a)|\right]+1
$$

and $[r]$ denotes the Gaussian integer of $r(r \in \mathbb{R})$.

\section{Some inequalities for random variables}

Throughout this section, let $0<a<b, r \in \mathbb{R}$, and let $X$ be a continuous random variable having the continuous probability density function $g:[a, b] \rightarrow \mathbb{R}$ with $g(t)>0$ on $(a, b), h:[a, b] \rightarrow \mathbb{R}$ with $h^{\prime}(t)=g(t)$ for $t \in(a, b)$ and the $r$-moment $E_{r}(X):=\int_{a}^{b} t^{r} g(t) d t$, which is assumed to be finite.

THEOREM 3. We have the inequalities

$$
\begin{aligned}
\left|E_{r}(X)-\frac{a^{r}+b^{r}}{2}\right| & \leq \frac{1}{2}\left(b^{r}-a^{r}\right)+\int_{a}^{b} \operatorname{sgn}\left(h^{-1}\left(\frac{1}{2}\right)-t\right) t^{r} g(t) d t \\
& \leq \frac{1}{2}\left(b^{r}-a^{r}\right) \quad \text { as } r \geq 0
\end{aligned}
$$


and

$$
\begin{aligned}
\left|E_{r}(X)-\frac{a^{r}+b^{r}}{2}\right| & \leq \frac{1}{2}\left(a^{r}-b^{r}\right)+\int_{a}^{b} \operatorname{sgn}\left(t-h^{-1}\left(\frac{1}{2}\right)\right) t^{r} g(t) d t \\
& \leq \frac{1}{2}\left(a^{r}-b^{r}\right) \text { as } r<0,
\end{aligned}
$$

respectively.

PROOF. If we put $f(t)=t^{r}(t \in[a, b]), h(t)=\int_{a}^{t} g(x) d x(t \in[a, b])$ and $x=(h(a)+h(b)) / 2=1 / 2$ in Corollary 1 , then we obtain (4.1) and (4.2). This completes the proof.

The following corollary is a special case of Theorem 3 .

COROLlaRY 3. The inequalities

$$
\left|E(X)-\frac{a+b}{2}\right| \leq \frac{b-a}{2}+\int_{a}^{b} \operatorname{sgn}\left(h^{-1}\left(\frac{1}{2}\right)-t\right) \operatorname{tg}(t) d t \leq \frac{b-a}{2}
$$

hold, where $E(X)$ is the expectation of the random variable $X$.

\section{Inequalities for the Beta and Gamma functions}

The following two functions are well known in the literature as the Beta function

$$
B(x, y):=\int_{0}^{1} t^{x-1}(1-t)^{y-1} d t, \quad x>0, y>0
$$

and the Gamma function,

$$
\Gamma(x):=\int_{0}^{\infty} e^{-t} t^{x-1} d t, \quad x>0,
$$

respectively.

The following inequality is an application of Theorem 1 for the Beta function.

THEOREM 4. Let $p, q>0$. Then we have the inequality

$$
\begin{aligned}
|B(p+1, q+1)-x| & \leq x+\int_{a}^{b} \operatorname{sgn}\left[t-((p+1) x)^{1 /(p+1)}\right] t^{p}(1-t)^{q} d t \\
& \leq x+\left(\frac{1}{p+1}-2 x\right)\left[1-((p+1) x)^{1 /(p+1)}\right]^{q} \\
& \leq \frac{1}{2(p+1)}+\left|x-\frac{1}{2(p+1)}\right|
\end{aligned}
$$

for all $x \in[0,1 /(p+1)]$. 
PROOF. If we put $a=0, b=1, f(t)=(1-t)^{q}, g(t)=t^{p}$ and $h(t)=t^{p+1} /(p+1)$ $(t \in[0,1])$ in Theorem 1, we obtain inequality (5.1) for all $x \in[0,1 /(p+1)]$.

The following remark is an application of Theorem 4 for the Gamma function.

REMARK 5. As $B(p+1, q+1)=\Gamma(p+1) \Gamma(q+1) / \Gamma(p+q+2)$, inequality (5.1) is equivalent to

$$
\begin{aligned}
\left|\frac{\Gamma(p+1) \Gamma(q+1)}{\Gamma(p+q+2)}-x\right| & \leq x+\int_{a}^{b} \operatorname{sgn}\left[t-((p+1) x)^{1 /(p+1)}\right] t^{p}(1-t)^{q} d t \\
& \leq x+\left(\frac{1}{p+1}-2 x\right)\left[1-((p+1) x)^{1 /(p+1)}\right]^{q} \\
& \leq \frac{1}{2(p+1)}+\left|x-\frac{1}{2(p+1)}\right|,
\end{aligned}
$$

that is,

$$
\begin{aligned}
|(p+1) \Gamma(p+1) \Gamma(q+1)-x(p+1) \Gamma(p+q+2)| \\
\quad \leq\left[x+\int_{a}^{b} \operatorname{sgn}\left[t-((p+1) x)^{1 /(p+1)}\right] t^{p}(1-t)^{q} d t\right](p+1) \Gamma(p+q+2) \\
\quad \leq\left[x+\left(\frac{1}{p+1}-2 x\right)\left[1-((p+1) x)^{1 /(p+1)}\right]^{q}\right](p+1) \Gamma(p+q+2) \\
\quad \leq\left[\frac{1}{2}+\left|x(p+1)-\frac{1}{2}\right|\right] \Gamma(p+q+2)
\end{aligned}
$$

and as $(p+1) \Gamma(p+1)=\Gamma(p+2)$, we get

$$
\begin{aligned}
\mid \Gamma(p & +2) \Gamma(q+1)-x(p+1) \Gamma(p+q+2) \mid \\
& \leq\left[x+\int_{a}^{b} \operatorname{sgn}\left[t-((p+1) x)^{1 /(p+1)}\right] t^{p}(1-t)^{q} d t\right](p+1) \Gamma(p+q+2) \\
& \leq\left[x+\left(\frac{1}{p+1}-2 x\right)\left[1-((p+1) x)^{1 /(p+1)}\right]^{q}\right](p+1) \Gamma(p+q+2) \\
& \leq\left[\frac{1}{2}+\left|x(p+1)-\frac{1}{2}\right|\right] \Gamma(p+q+2)
\end{aligned}
$$

for any $x \in[0,1 /(p+1)]$.

\section{References}

[1] T. M. Apostol, Mathematical Analysis, 2nd ed. (Addison-Wesley, Reading, Mass.-London-Don Mills, Ont., 1975). 
[2] N. S. Barnett, S. S. Dragomir and C. E. M. Pearce, "A quasi-trapezoid inequality for double integrals", ANZIAM J. 44 (2003) 355-364.

[3] P. Cerone and S. S. Dragomir, "Trapezoidal type rules from an inequalities point of view", in Handbook of Analytic-Computational Methods in Applied Mathematics (ed. G. Anastassiou), (CRC Press, New York, 2000) 65-134.

[4] P. Cerone, S. S. Dragomir and C. E. M. Pearce, "A generalized trapezoid inequality for functions of bounded variation", Turkish J. Math. 24 (2000) 147-163.

[5] S. S. Dragomir, "On the trapezoid quadrature formula for Lipschitzian mappings and applications", Tamkang J. Math. 30 (1999) 133-138.

[6] S. S. Dragomir, "On the trapezoid quadrature formula and applications", Kragujevac J. Math. 23 (2001) 25-36.

[7] S. S. Dragomir, P. Cerone and A. Sofo, "Some remarks on the trapezoid rule in numerical integration", Indian J. Pure Appl. Math. 31 (2000) 475-494.

[8] S. S. Dragomir and T. C. Peachey, "New estimation of the remainder in the trapezoidal formula with applications", Stud. Univ. Babes-Bolyai Math. (Cluj) 45 (2000) 31-42.

[9] L. Fejér, "Uberdie Fourierreihen, Il", Math. Natur. Ungar. Akad. Wiss. 24 (1906) 369-390. 